\title{
Tidal effects on the spatial structure of the Local Group
}

\author{
S. Pasetto ${ }^{1,2}$ and C. Chiosi ${ }^{3,4}$ \\ 1 Astronomisches Rechen-Institut, Zentrum für Astronomie der Universität Heidelberg, Mönchhofstr. 12-14, 69120 Heidelberg, \\ Germany \\ e-mail: spasetto@ari.uni-heidelberg.de \\ 2 Max Planck Institut für Astronomie, Königstuhl 17, 69117 Heidelberg, Germany \\ 3 Department of Astronomy, Padova University, Vicolo dell'Osservatorio 2, 35122 Padova, Italy \\ e-mail: cesare.chiosi@unipd.it \\ 4 Max Planck Institut für Astrophysik, Karl-Schwarzschild-Str. 1, 85748 Garching, Germany
}

Received 15 October 2008 / Accepted 18 February 2009

\section{ABSTRACT}

\begin{abstract}
Aims. The spatial distribution of galaxies in the Local Group (LG) is the footprint of its formation mechanism and the gravitational interactions among its members and the external massive galaxies or galaxy groups. Using a 3D-geometrical description of the spatial distribution of all the members of the LG (not only the satellites of the MW and M31) based on present-day data of positions and distances, we found in our previous study that all galaxies (MW, M31, their satellites, and even the most distant objects) are confined within a slab of about $200 \mathrm{kpc}$ thickness. Examining how external galaxies or groups would gravitationally affect (and eventually alter) the planar structure (and its temporal evolution) of the LG, they found that the external force field acts parallel to the plane determined by geometry and studied this with the Least Action Principle.

Methods. In this paper, we thoroughly investigated the role played by the tidal forces exerted by external galaxies or galaxy groups on the LG galaxies (the most distant dwarfs in particular) in shaping their large-scale distribution. We studied in particular an idea based on the well-known effect of tidal interactions, according to which a system of mass-points can undergo not only tidal stripping but also tidal compression and thus become flatter.

Results. Excluding the dwarf galaxies tightly bound to the MW and M 31, the same tidal forces can account for the planar distribution of the remaining dwarf galaxies. We analytically recover our previous results and prove that a planar distribution of the LG dwarf galaxies is compatible with the external force field. We also highlight the physical cause of this result.
\end{abstract}

Key words. galaxies: Local Group - galaxies: dwarf - galaxies: kinematics and dynamics

\section{Introduction}

Over the years, many attempts have been made to find the spatial distribution of the LG galaxies. Limiting ourselves to a few pioneering studies Kahn \& Woltjer (1959) and Raychaudhury \& Lynden-Bell (1989) suggested a planar distribution based on studies of the LG dynamics; Hartwick (2000) found a flat ellipsoid with axial ratios $(a, b, c)=(1.00,0.51,0.19)$, which does not differ too much from a plane; and finally Kunkel (1979), Grebel et al. (1999), and Fusi Pecci et al. (1995) suggested that the satellite dwarf galaxies of the MW and M 31 lie on planes ${ }^{1}$.

Kroupa et al. (2005) and Metz et al. (2007) suggested the satellites of the MW occupied a planar distribution, which however could also be explained as a consequence of the distribution of sub-haloes (Zentner et al. 2005) in the early cosmological stages (Kang et al. 2005). The same problem was investigated by Koch \& Grebel (2006) for the satellites of M 31 with similar conclusions.

\footnotetext{
${ }^{1}$ Closely related to this problem is the issue of the anisotropic distribution of inner sub-haloes with respect to larger haloes in relation to the Holmberg effect (Holmberg 1969) with dissimilar results, e.g. Sales \& Lambas (2004), Yang et al. (2006). What matters here (and is still debated) is whether disruptions and tidal effects can create the apparent polar alignment of the dwarf satellites around the host galaxy or, for the particular case of the LG, the position of the dwarf galaxies is the consequence of peculiar directions of pre-existing cosmological filaments.
}

Starting from the basic idea that an off-center hydrodynamical collision occurred some $10 \mathrm{Gyr}$ ago between the primordial gas-rich M 31 galaxy and the MW, and compressed the halo gas to form all the LG dwarf galaxies, Sawa \& Fujimoto (2005) suggested that the new-born dwarf galaxies would be located near the orbital plane of the MW and M31. They argued that this view is also consistent with their visual inspection of the 2D sky distribution of the LG members and that a well-defined plane of finite thickness is found, within which most of the member galaxies are confined.

Pasetto \& Chiosi (2007, hereafter PC07) attacked the problem from a completely different perspective. In summary, adopting known positions and distances and using analytical geometry, they looked for the plane that minimizes the distances of all galaxies in the LG with respect to it (not only the MW and M 31 and their satellites, but also the distant dwarfs). The second part of their study was to find a dynamical justification of the planar distribution. To this aim, they applied the Hamilton Method (Minimum Action) to investigate the dynamics of the LG complex and the action of the gravitational forces exerted by external nearby galaxies or groups. They found that the planar distribution is fully compatible with the minimum action and that the external force field is probably compatible within the plane determination. This field would then pull the LG galaxies along, without altering their planar distribution. Special care was taken to evaluate the robustness of the result. 
To explain the different results obtained by Kroupa et al. (2005), Koch \& Grebel (2006), and Sawa \& Fujimoto (2005), we note that the various studies did not use identical galaxy samplings, they did not start from the same working physical hypotheses nor they deal with the same dynamical regime. In brief:

(i) the planes for the MW and M 31 satellites (Kroupa et al. 2005; Koch \& Grebel 2006, respectively) are of a local nature because they are the consequence of strong collisional dynamics with the host galaxy (hereafter HG). No easy explanation can be found as to why these planes survive for long time (more than a few dynamical time scales) due to the peculiar proper motions of the dwarfs that determine these planes (see, e.g., the ideas in Lynden-Bell 1983; Lynden-Bell \& Lynden-Bell 1995; Palma et al. 2002; applied then by Metz et al. 2008). The role of distant dwarfs in determining the structure of the whole LG is not considered. Therefore, these planes cannot be extended to the whole LG;

(ii) in Sawa \& Fujimoto (2005), the solution for a common plane is based on the ad hoc initial hypothesis concerning the origin of the angular momentum. The sample of dwarf galaxies used to determine the plane is limited to the satellites of the two HGs. Finally, the orbits of these dwarfs are constrained to lie on this plane;

(iii) in $\mathrm{PC} 07$, the common plane is chosen by assuming that it contains the MW and M31 and minimizing the distances of all remaining LG galaxies to this plane, including also the distant ones. This can be justified by considering that the HG satellites are strongly influenced by local dynamics (with continuous modification of their orbits, including possible captures by the HGs) and that if a common planar distribution for all LG galaxies exists, this should evidenced by the more external galaxies. They are much less likely to be affected by strong interactions with one of the HGs and therefore more sensitive to the influence of external galaxies and/or groups. The plane found by PC07 is a slab of about $200 \mathrm{kpc}$ thickness, i.e., the largest apo-center of the HG satellites most probably falls inside this slab.

In addition, $\mathrm{PC} 07$ showed that the external force field runs parallel to their plane. It is likely that among the galaxies of the LG those that feel the external action the most are the dwarfs not tightly bound to any HG. In other words, this group of dwarfs could act as a tracer of the external force field. Our aims is to find a gravitational action that is able to induce, on a long timescale, a sort of extended slab. Tidal forces are known to engender this kind of response.

The main goal of this study is to highlight the physical nature of the results obtained by PC07, which had a rather complicated dynamical description requiring a numerical approach. We therefore develop a simpler linear approximation that is much easier to handle and yet able to provide a physical insight.

The plan of the paper is as follows. In Sect. 2, we define the tidal-force field acting on the whole LG. The tidal forces are those developed by external groups of galaxies. We combine the MW with its satellites (M31 and its satellites) for which a suitable treatment is required and look at the remaining dwarf galaxies. On a long timescale, the external tidal forces can engender a planar distribution of these dwarf galaxies in the LG. In Sect. 3, we go deeper into this issue and check whether the planar distribution is a mere coincidence or the consequence of fundamental laws of mechanics. The answer is the latter: the planar distribution corresponds to a minimum energy and stable configuration of the whole system. The dwarf galaxies must lie on a plane because of the long timescale influence of the tidal forces exerted by massive galaxies or galaxy groups external to the LG. Furthermore, the plane found by PC07 and the minimum energy plane are coincident and the situation is stable. Finally, in Sect. 4 we summarize our results and present some general considerations.

\section{LG structure and tidal forces}

Looking at the composition of the LG, three main components can be identified: two massive galaxies (MW and M 31), their respective groups of bounded satellites, and a large number of distant dwarfs galaxies loosely interacting or even uncoupled to the dominant galaxies. As far as the gravitational interaction is concerned, there are two questions we are interested in addressing:

(1) How does the external force field change with time?

(2) Have the dwarf galaxies that are not members of the MW or the M 31 family been affected by the tidal interaction with the external force field?

First, to obtain an approximate estimate of the influence of the tidal forces acting on the LG, we had to develop an accurate geometrical description of the LG during its temporal evolution. Although the tidal forces are weak, they can produce a cumulative effect of compression that could explain the planar distribution by acting over a long time. However, if this action is not always pointing in the same direction, the net effect can be small, and the tidal effect can no longer be the physical mechanism compressing the dwarf galaxy distribution. To follow the direction of a tidal field, we applied the usual formalism of the tidal tensor (e.g., Misner et al. 1973, Chap. 1) to all galaxies of the LG and attempt to understand its global behavior under the action of the external force field.

Frame of reference. The true mutual interaction between the MW and M31 suggests that they can be considered as a privileged system, whose center of mass $(\mathrm{CM})$ can be assumed to be the origin of a reference frame (presented in Fig. 1 and described in more detail below). The positions and motions of any other dwarf in the LG that is not a member of the MW and M 31 families can be given relative to this CM. This is reminiscent of the geometry of the restricted three-body problem (e.g., Szebehely 1967) but here the time evolution of the CM will be followed. In particular, the stationary action principle was applied in PC07 to produce a possible solution for the motion of the external groups acting gravitationally on the LG as well as MW and M 31 (their Table 4). From this solution, we can infer the spatial evolution in the time, $t$, of the entire LG-barycentric system $\boldsymbol{x}^{\mathrm{CM}_{\mathrm{LG}}}=\boldsymbol{x}^{\mathrm{CM}_{\mathrm{LG}}}(t)$, which is nearly coincident with the center of mass of the MW + M 31 system.

We started by considering the general expression for the external tidal tensor as function of any potential $\Phi$ expressed in the reference system $S_{0}$ of Fig. 1, to describe the external tidal tensor acting on the complex MW+M 31

$$
\begin{aligned}
T_{i j}\left(\boldsymbol{x}^{\mathrm{CM}_{\mathrm{LG}}}\right)= & \sum_{g \neq \mathrm{MW}, \mathrm{M} 31} \frac{G M_{g}}{\left\|x_{i}^{\mathrm{CM}}-x_{i}^{g}\right\|^{3}} \\
& \times\left(\frac{3\left(x_{i}^{\mathrm{CM}}-x_{i}^{g}\right)\left(x_{j}^{\mathrm{CM}}-x_{j}^{g}\right)}{\left\|x_{i}^{\mathrm{CM}}-x_{i}^{g}\right\|^{2}}-\delta_{i j}\right)
\end{aligned}
$$




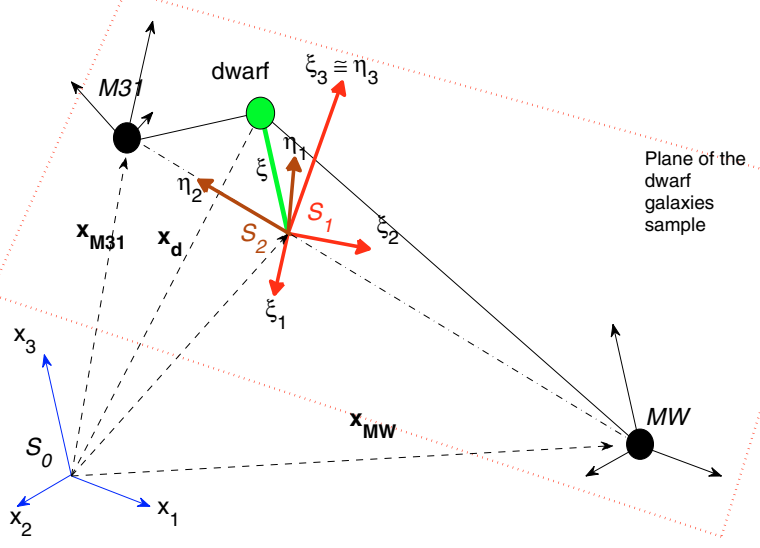

Fig. 1. Sketch of the geometrical framework we have adopted. First, we define the inertial reference frame, always named $S_{0}$, with axis $\left(S_{0}, x_{1}, x_{2}, x_{3}\right)$. It is centered on the $\mathrm{CM}$ of the external galaxy groups listed in Table 1 . We then introduce two auxiliary reference frames: the first centered on the barycenter of the MW and M31, which is aligned with the principal axes of the eigensystem provided by Eq. (1). This system is called $S_{1}$ and has axes $\left(\xi_{1}, \xi_{2}, \xi_{3}\right)$ with $\xi_{3}$ pointing roughly in the direction orthogonal to the plane of the dwarf galaxies (red dotted rectangle). The second is called $S_{2}$ and it has axes $\left(\eta_{1}, \eta_{2}, \eta_{3}\right)$ with $\eta_{3}$ pointing in the same direction of $\xi_{3}$, and $\eta_{2}$ pointing in the direction of M31; see the text for details. The current position vector of a generic dwarf galaxy in the system $S_{1}$ and $S_{2}$ is called $\boldsymbol{\xi}$ and $\boldsymbol{\eta}$ respectively (e.g. we show $\xi$ in the figure). It is always oriented toward the generic dwarf galaxy.

Table 1. External galaxy groups gravitationally influencing the LG dynamics.

\begin{tabular}{lrrccr}
\hline \hline $\begin{array}{l}\text { Group } \\
\text { Name }\end{array}$ & $\begin{array}{l}l \\
\circ\end{array}$ & $\begin{array}{r}b \\
\circ\end{array}$ & $\begin{array}{c}d^{b} \\
\mathrm{Mpc}\end{array}$ & $\begin{array}{c}M \\
10^{12} M_{\odot}\end{array}$ & $\begin{array}{r}V_{r}{ }^{c} \\
\mathrm{~km} \mathrm{~s}^{-1}\end{array}$ \\
\hline IC 342 & 138.2 & 10.6 & 3.3 & 12.6 & 171.0 \\
Maffei & 136.4 & -0.4 & 3.5 & 6.3 & 152.0 \\
M 81 & 142.1 & 40.9 & 3.7 & 1.6 & 130.0 \\
Cen A & 309.5 & 19.4 & 2.7 & 4.7 & 371.0 \\
Sculptor & 105.8 & 85.8 & 3.2 & 6.3 & 229.0 \\
M 83 & 312.1 & 25.9 & 4.5 & 0.8 & 249.0 \\
\hline Andromeda & 121.21 & -21.60 & 0.76 & 3.16 & -123.00 \\
Milky way & 0 & 0 & 0.00 & 2.20 & 0.00 \\
\hline
\end{tabular}

${ }^{a}$ Each group is indicated by the dominant galaxy. The members of each group are the same as in PC07 to whom the reader should refer for all details (their Sect. 4 and Table 2). To this list the MW and M31 are added. ${ }^{b}$ The uncertainties in the distances and radial velocities are omitted. ${ }^{c}$ The radial velocities are quoted relative to the center of the MW. No correction for the motion of the Sun toward the Local Standard of Rest is applied because it falls below the accuracy adopted in this study.

where $G$ is the gravitational constant, $x^{g}$ are the coordinates of the external galaxy groups of Table 1 of mass $M_{g}, \delta$ is the Dirac's function, and $\|$.$\| is the standard norm. We evaluated the tidal$ tensor at the barycenter of the LG, $x^{\mathrm{CM}_{\mathrm{LG}}}=\boldsymbol{x}^{\mathrm{CM}_{\mathrm{LG}}}(t)$ as defined above but with the time dependence being omitted.

To obtain an idea of the effects we are looking for, we make the following preliminary considerations. We assume that the most distant dwarfs defining the plane $\pi$ discovered by PC07 have positions that are determined, in their initial conditions (e.g., 9 Gry ago), by the tidal tensor of external objects. We then expect the total tidal tensor (TTT), $T_{i j}^{\text {tot }}(\boldsymbol{x})$, defined by Eq. (1) including in the sum also the MW and M31, to give, if reduced to its normal form, a single most negative eigenvalue. Between the three eigenvectors of TTT, the one corresponding to this most negative eigenvalue will indicate the direction of the tidal compression (see classical textbooks such as Misner et al. 1973, Chap. 1; Binney \& Tremaine 1987, Chap. 7; or some applications as in Raychaudhury \& Lynden-Bell 1989). Thus, if we also find this behavior in our TTT evaluated at the position of a dwarf galaxy far away from the MW or M31, this could imply that the compression does indeed occur. For example, a simple case would be that a dwarf presently belonging to the plane $\pi$ was also formed by some mechanism in the plane $\pi$ or close to it. In this case we can hypothesize that the tidal force acting on this dwarf should be similar to the tidal force that acted on the plane in the past, say 9 Gyr ago. We can estimate these eigenvalues and their eigenvector directions by combining the equations of the plane $\pi$ (see Eq. (2)), obtained by considering the current dwarf galaxy distribution, and Eq. (1), which can also be evaluated backwards in time. If we find compatible values between different points at different epochs, then we can claim that the effect we are searching for may have occurred.

If the plane $\pi$ is a slab with a diameter of $4 \mathrm{Mpc}$ and $200 \mathrm{kpc}$ thick, we can evaluate the TTT at any point on this plane $P \in \pi$, say $2 \mathrm{Mpc}$ away from the barycenter of the LG, $T_{i j}^{\mathrm{tot}}\left(x^{P}\right)$. The resulting eigenvalue of this tidal tensor, e.g., 9 Gyr ago, is $\lambda_{9 \mathrm{Gyr}}^{P}=\{0.024,-0.016,-0.007\}$. The same evaluation can then be repeated for the barycenter position $T_{i j}^{\mathrm{tot}}\left(x^{\mathrm{CM}_{\mathrm{LG}}}\right)$, obtaining $\lambda_{9 \mathrm{Gyr}}^{\mathrm{CM}_{\mathrm{LG}}}=\{0.022,-0.014,-0.007\}$. This result strongly suggests that in the past the force determining the subsequent orbital evolution of a generic dwarf had a component that squeezed the motion toward the plane. Proceeding in this way, we can prove the compatibility of the eigenvalues of the TTT for every position on the plane $\pi$, i.e., $T_{i j}^{\text {tot }}\left(x^{\mathrm{CM}_{\mathrm{LG}}}\right) \cong T_{i j}^{\mathrm{tot}}\left(x^{P}\right) \forall P \in \pi$. This clearly allows us to explore the possibility that the plane $\pi$ of PC07 is produced by the tidal forces acting on the LG during a large fraction of the Hubble time, i.e., we want to extend this estimation not only to the present time $t=t_{0}$ but also to the time $e^{2} t<t_{0}$.

To proceed further, we need to search the eigenvectors associated with the tidal tensor that have the most negative eigenvalues. In the limits of our approximation, they should retain a direction with respect to an inertial reference frame not too far from the normal to the geometrically plane $\hat{\boldsymbol{n}}(\pi)$ for most of the Hubble time, which can nowadays be inferred by simple inspection of the dwarf galaxies' distribution in the LG. To prove this, we solve the eigensystem for the tidal tensor of Eq. (1) as a function of time. The solutions are presented in Table 2: the three eigenvalues are in Cols. (2) to (4) and the corresponding eigenvectors are in Cols. (5) through (13). The results in which we are interested are limited in time to a range where monotonicity of the trend of the eigenvalues can be exploited to reveal an integrated cumulative effect of compression or expansion. We find that our range of interest must cover the past $9 \mathrm{Gyr}$, imposing a lower limit to our analysis of $t=t_{\text {inf }} \cong 9 \mathrm{Gyr}$. Before this $t_{\text {inf }}$, the configuration of the eigenvalues differs slightly. From Table 2, we see that the time evolution of the eigenvalues shows a phase with one compressive direction compared to two positive expansion directions, and before that, as well as after $t=t_{\text {inf }}$, we see

2 Of course a more correct computation could have been performed by knowing distribution of the dwarf galaxies in the past, but unfortunately we cannot track back the past orbits of the dwarf galaxies belonging to the plane $\pi$ today; therefore Eq. (2) for the plane $\pi$ cannot be directly determined in its time evolution. 
Table 2. Temporal evolution of the eigenvalues and eigenvectors as a function of the look-back time $t_{\mathrm{lb}}$ in Gyr.

\begin{tabular}{crrrrrrrrrrrr}
\hline \hline$t_{\mathrm{lb}}$ & $\lambda_{1}$ & $\lambda_{2}$ & $\lambda_{3}$ & $n_{x}^{\left(\lambda_{1}\right)}$ & $n_{y}^{\left(\lambda_{1}\right)}$ & $n_{z}^{\left(\lambda_{1}\right)}$ & $n_{x}^{\left(\lambda_{2}\right)}$ & $n_{y}^{\left(\lambda_{2}\right)}$ & $n_{z}^{\left(\lambda_{2}\right)}$ & $n_{x}^{\left(\lambda_{3}\right)}$ & $n_{y}^{\left(\lambda_{3}\right)}$ & $n_{z}^{\left(\lambda_{3}\right)}$ \\
\hline 13.3 & 0.0125 & -0.0049 & -0.0076 & 0.310 & 0.507 & 0.804 & 0.934 & -0.008 & -0.356 & 0.174 & -0.862 & 0.476 \\
12.3 & 0.0058 & 0.0001 & -0.0060 & -0.266 & 0.411 & 0.872 & 0.944 & 0.292 & 0.151 & 0.192 & -0.864 & 0.465 \\
11.0 & 0.0031 & 0.0008 & -0.0038 & 0.941 & 0.052 & -0.334 & 0.265 & 0.498 & 0.826 & 0.209 & -0.866 & 0.455 \\
9.5 & 0.0030 & -0.0002 & -0.0028 & 0.971 & 0.235 & -0.043 & -0.066 & 0.436 & 0.897 & 0.230 & -0.868 & 0.439 \\
7.9 & 0.0030 & -0.0006 & -0.0024 & 0.954 & 0.297 & 0.026 & -0.146 & 0.390 & 0.909 & 0.259 & -0.871 & 0.416 \\
6.2 & 0.0030 & -0.0007 & -0.0023 & 0.939 & 0.341 & 0.045 & -0.171 & 0.346 & 0.922 & 0.299 & -0.873 & 0.384 \\
4.6 & 0.0033 & -0.0008 & -0.0024 & 0.919 & 0.391 & 0.050 & -0.175 & 0.292 & 0.940 & 0.353 & -0.873 & 0.336 \\
3.0 & 0.0037 & -0.0009 & -0.0028 & 0.887 & 0.458 & 0.059 & -0.171 & 0.208 & 0.963 & 0.429 & -0.864 & 0.263 \\
1.4 & 0.0046 & -0.0010 & -0.0036 & -0.829 & -0.551 & -0.097 & -0.158 & 0.065 & 0.985 & 0.536 & -0.832 & 0.141 \\
0.0 & 0.0065 & -0.0017 & -0.0047 & -0.738 & -0.657 & -0.151 & -0.064 & -0.154 & 0.986 & 0.671 & -0.738 & -0.071 \\
\hline
\end{tabular}

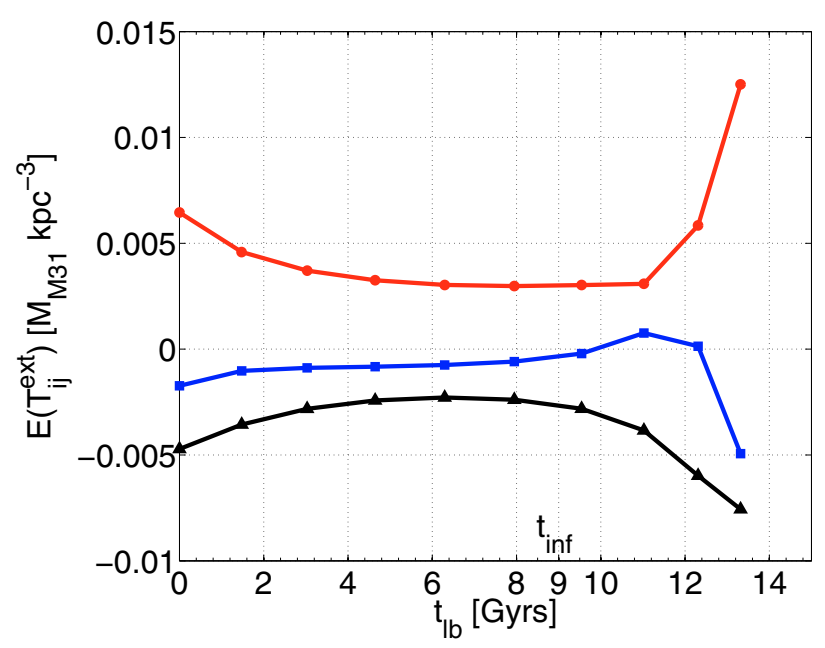

Fig. 2. Evolution of the eigenvalues $E\left(T_{i j}^{\text {ext }}\right)$ of Table 2 as a function of the look-back time in Gyr (Col. 1). The red line with dots is for the first positive eigenvalue $\lambda_{1}$, the blue line with squares is the second eigenvalue $\lambda_{2}$ negative for $t_{\mathrm{lb}}<9.5$ Gys, and the black with triangles is for the third (most negative) eigenvalue $\lambda_{3}$.

two negative directions compared with one positive. For simplicity, we do not be treat analytically these switchings between the configurations. We are only interested in the last most-dominant time-evolution of the monotonic behavior of the eigenvalues. Since we exclude the first three rows of Table 2 (the primordial evolution prior to $t_{\text {inf }}$ ) from our analysis, from now on, we uniquely identify (unless otherwise specified) with $\lambda_{1}, \lambda_{2}$, and $\lambda_{3}$, the positive eigenvalue, the second negative eigenvalue, and the most negative eigenvalue, respectively, for the eigenvectors of the external tidal tensor defined in Eq. (1) followed in their time $t$ evolution exclusively for $\left.t \in] t_{\text {inf }}, t_{0}\right]$. The most negative eigenvalue (Col. 4) and its evolution in the past 9 Gyr and projections of the associated eigenvector onto the axes of the inertial system are highlighted in italics. The time variations in the three eigenvalues are shown in Fig. 2.

From the data displayed in Fig. 2, it is evident that one of the negative eigenvalues dominates. Therefore, the external field acting on the LG may produce the planar distribution of the dwarf galaxies. This is an interesting result because:

1. it proves that, using the tidal tensor, we can obtain analytically the same results as $\mathrm{PC} 07$ for the behavior of the external field. The external force field turns out to be compatible with the dwarf galaxies being in a flat spatial distribution that remains constant for a large fraction of the Hubble time. Moreover, we can argue that the external force field had

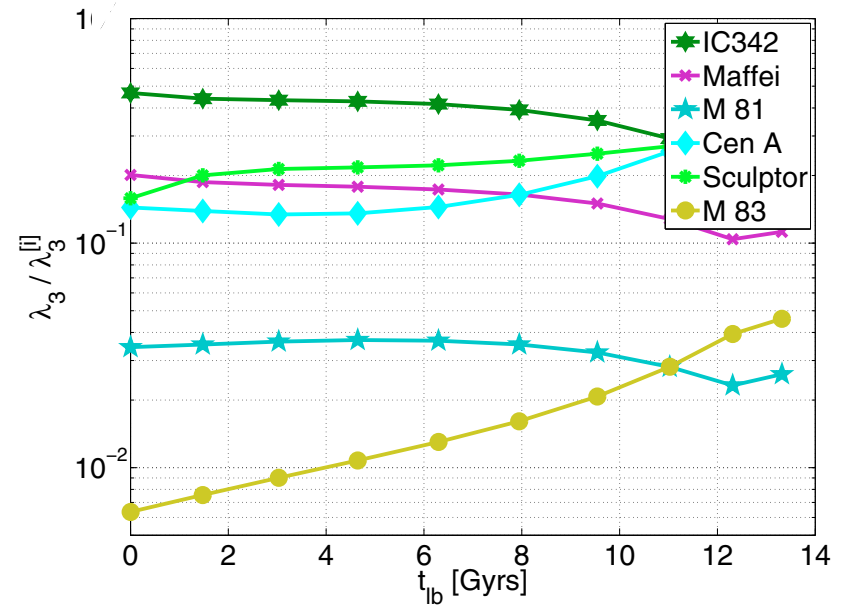

Fig. 3. Here we tracked back the influence of each individual component in the sum of Eq. (1) for the most negative eigenvalue, $\lambda_{3}^{[i]}$. The curves are normalized to the black line, $\lambda_{3}$, of Fig. 2 (see text for details). The major contribution overall is dominated by the IC342 group, followed by Cen A, Maffei and Sculptor groups. The contribution of M 83, even if nowadays marginal, could have played a role in the past in the imprint of the initial condition of the LG dwarf galaxies.

already started to flatten the spatial distribution of the dwarf galaxies prior to $t_{\text {inf }}$. If we investigate the relevance of the different groups on this flattening effect in more detail, we can plot in Fig. 3 the normalized trend of the most negative eigenvalues of Eq. (1) divided into its components. Here we see the most negative eigenvalues of the sum in Eq. (1) normalized to the overall sum (hence the black line in Fig. 2 represents the unitary constant upper bound to the figure). As we can infer from the figure, the influence of the IC342 group has always been the most significant, followed by the effects of Maffei, Sculptor, and Cen A groups. This is expected from their masses and positions listed in Table 1 . The time evolution shown in this Figure confirms their relative importance in the compressing effect on the LG for its temporal evolution. The contribution of the M 83 group is slightly less important, although is was nevertheless as important as that of the M81 group 11 Gyr ago;

2. we must clarify once and for all that the present result does not prove that the spatial distribution of dwarf galaxies in the LG must be flat, but only that the external force field is compatible with such a flat distribution;

3. the external force field acting only on the two main HGs (MW and M 31) of the LG is compatible with a flat spatial distribution of the distant dwarfs, but this does not provide 


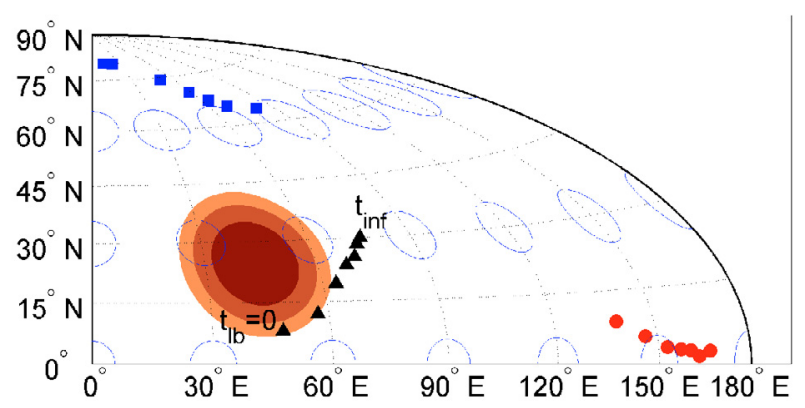

Fig. 4. The Hammer projection of the sky position of the normal to the plane found by PC07 (from purely geometrical arguments). The color code from light red to dark brown corresponds to the $3 \sigma, 2 \sigma$, and $1 \sigma$ uncertainties as estimated by PC07 from the principal component analysis and projected onto the sky. The shape of the three shaded areas becomes more oblate at increasing angular direction $b$. The classical Tissot's circles, perfect circles of angular radius of $7 \mathrm{deg}$ have been plotted to help visualize the angular distances. We have used the same symbols and time intervals as in Fig. 2 and Table 2. The black triangles nearly overlap the shaded area, showing that the direction of the eigenvector with the most negative eigenvalue lies close to the direction of the normal to the PC07 plane, which is of course constant in the figure because it is derived from the observational data available today. For comparison we also show the evolution of the other two orthogonal eigenvectors indicated by the same symbols as in Fig. 2.

any constraint on the distribution of the nearby dwarf satellites around their HG (both MW and M31);

4. furthermore, the planar distribution detected in the geometrical analysis of PC07 cannot a priori be related to the planar distribution suggested by the compression effect described by the tidal tensor affecting the LG. The subject of the following analysis is to explain the coincidence claimed by PC07 between the geometrical and dynamical planes.

We now seek to prove that the following two issues are tightly related: (i) There exists a plane in the spatial distribution of the LG dwarf galaxies that is expected because of the external tidal force acting on the LG; (ii) in the context of the linear approximation that we adopted (see below), the tidal-force field compatible with a flat distribution has an orientation whose normal vector is aligned with the normal vector of the planar distribution $\pi$ found by PC07.

The equation for the plane $\pi$ can be rewritten here in the reference frame $S_{0}$ as

$0.64 x_{1}-0.61 x_{2}-0.45 x_{3}=0$

with a direction $(l, b)=\left(45^{\circ}, 27^{\circ}\right)$ for $\hat{\boldsymbol{n}}(\pi)$, the normal vector to the plane. Furthermore, the directions of the three basis vectors $\hat{\boldsymbol{e}}_{\mathrm{S}_{0}}$ in which the eigenvalues of Table 2 are expressed were chosen to be collinear with those of the reference frame adopted by PC07 (we assumed that the Sun is located at the center of $\mathrm{MW}$, and the error of $\approx 8.5 \mathrm{kpc}$ is negligible in the present context). Therefore, we can assume that only at the present time $t_{0}$ does the reference system in which the above equation of the plane is written have orthonormal vectors parallel to the basis vector of reference system adopted at $t=t_{0}$. It follows that the Hammer projection from the center of mass of the LG shows the eigenvector directions as a function of time and the normal vector to the geometrical plane of PC07 are as in Fig. 4.

Interestingly, it is the eigenvector relative to the most negative eigenvalue that lies closer to the direction of the normal of the plane of the dwarf galaxies.
Considering that every point in this map has an error radius of about $\pm 9^{\circ}$, inherited from the Minimum Action analysis, and considering the uncertainty in the angular definition of the direction of $\hat{\boldsymbol{n}}(\pi)$, the normal to the geometrical plane and the direction of the vector associated with the most negative eigenvalue are compatible at the present time at the $3 \sigma$-level of confidence. Moreover, the eigenvector of the most negative eigenvalue $\lambda_{3}$ of the tidal tensor moves, maintaining a direction not so far from the direction that we can nowadays deduce from the observation for $\hat{\boldsymbol{n}}(\pi)$. This result, which was already present in PC07, is recovered here in a semi-analytical treatment of the whole problem. The key question to be answered now is whether the coincidence is causal or casual and what the meaning of all this is.

\section{Causal or casual?}

The tidal tensor can be derived from the Taylor expansion of the force field (see next section). This implies that the object we want to investigate (a dwarf galaxy) is influenced by a smoothly varying potential in the course of its evolution.

The large-scale description of the gravitational interaction adopted here and by PC07 becomes inaccurate on the distance scales of the closer HG-dwarf satellite interactions where satellite dwarf galaxies experience kick-off, are continually absorbed into the halo of the HG (MW and M31 in our case) and the collisionless description is incorrect. A dwarf galaxy in these closer samples, which experiences a far stronger direct interaction with the $\mathrm{HG}$, does not respond significantly to the weaker external field acting on it. On the other hand, the collisionless description is suited to deal with the effects of distant galaxy groups (see the list in Table 1) and hence the influence of the external field on the other dwarf galaxies far away from a HG (see e.g., Raychaudhury \& Lynden-Bell 1989; Peebles 1990; Dunn \& Laflamme 1993; Peebles 1994).

To formalize out results in the previous section, we present the following two hypotheses, one about the directions of the eigenvectors and the other about their values:

1. we do not consider the evolutionary stages of the Universe older than $t_{\mathrm{lb}}>9 \mathrm{Gyr}$ (look-back time) when assuming a monotonic behavior of a single negative eigenvalue;

2. we assume that the eigenvector corresponding to the most negative eigenvalue always points in the same direction as the inertial reference frame in the non-comoving coordinate system.

The frame of reference. First, we define $S_{0}$ as the inertial reference frame centered on the CM of the external galaxy groups listed in Table 1. We also introduce a second reference frame $S_{1}$, which is non-inertial and comoving with the CM of the LG, and approximately coincident with the CM of the pair M 31 and MW. Moreover, we assume that the principal axes of this reference frame are always collinear with the principal axes of the external tidal tensor, $\boldsymbol{x}_{\mathrm{M} 31}$ is the coordinate vector of M 31 in $S_{0}$, $x_{\mathrm{MW}}$ is the coordinate vector of MW in $S_{0}, \boldsymbol{x}$ is the vector of a generic dwarf galaxy in $S_{0}$, and $\boldsymbol{R}$ is the position of the M31MW barycenter, i.e., the origin of $S_{1}$ in $S_{0}$. The generic position vector in the frame $S_{1}$ is $\boldsymbol{\xi}$, which is used to indicate the generic position of a dwarf galaxy. Both reference frames are orthogonal (see Fig. 1).

Equations of motion. We note only that the generic position vectors in the two reference frames are now written as $\boldsymbol{x}=\boldsymbol{x}_{\mathrm{LG}}^{\mathrm{CM}}+$ $\boldsymbol{O} \boldsymbol{\xi}=\boldsymbol{R}+\boldsymbol{O} \boldsymbol{\xi}$, where $\boldsymbol{O} \in S \boldsymbol{O}(3)$ is the generic rotation matrix with elements $O_{i j}$. In a similar way, for the time derivative of 
$\boldsymbol{x}$ we can write $\dot{\boldsymbol{x}}=\dot{\boldsymbol{x}}_{\mathrm{LG}}^{\mathrm{CM}}+\boldsymbol{O}(\dot{\boldsymbol{\xi}}+\boldsymbol{\Omega} \times \boldsymbol{\xi})$ or, in more compact notation

$\dot{\boldsymbol{x}}=\boldsymbol{V}+\boldsymbol{O}(\dot{\boldsymbol{\xi}}+\mathbf{\Omega} \times \xi)$,

where $\boldsymbol{\Omega}$ is the angular velocity and we put $\boldsymbol{V}=\dot{\boldsymbol{x}}_{\mathrm{LG}}^{\mathrm{CM}}$.

The time derivative of the velocity equation yields the equation of motion

$\ddot{\boldsymbol{x}}=\boldsymbol{A}+\boldsymbol{O}(\ddot{\boldsymbol{\xi}}+2 \mathbf{\Omega} \times \dot{\boldsymbol{\xi}}+\dot{\boldsymbol{\Omega}} \times \boldsymbol{\xi}+\boldsymbol{\Omega} \times(\mathbf{\Omega} \times \boldsymbol{\xi}))$,

where in the second equation the vector $\boldsymbol{A}$ has been used to simplify the notation for the acceleration $\ddot{x}_{\mathrm{LG}}^{\mathrm{CM}}$ of the barycenter. Moreover, to underline the physical meaning of the different terms we recall the previous equation and write

$\ddot{\boldsymbol{\xi}}=\boldsymbol{O}^{T}(\ddot{\boldsymbol{x}}-\boldsymbol{A})-2 \boldsymbol{\Omega} \times \dot{\boldsymbol{\xi}}-\dot{\boldsymbol{\Omega}} \times \boldsymbol{\xi}-\boldsymbol{\Omega} \times(\boldsymbol{\Omega} \times \boldsymbol{\xi})$.

From this last equation it is evident that the acceleration $\ddot{\xi}$, experienced by a dwarf galaxy in the non-inertial reference frame, is the sum of different terms due to the motion of the frames $S_{1}$ and $S_{0}$ and the fictional forces that appear due to the non-inertial nature of $S_{1}:-\boldsymbol{\Omega} \times(\boldsymbol{\Omega} \times \boldsymbol{\xi})$ is the centrifugal effect, $-2 \boldsymbol{\Omega} \times \dot{\boldsymbol{\xi}}$ is the Coriolis effect proportional to the velocity of the dwarf, and $-\dot{\boldsymbol{\Omega}} \times \boldsymbol{\xi}$ is the effect caused by the non-constant rotation rate of $S_{1}$. As usual, we assume that the rotation matrix linking the different orthonormal reference frames is $\boldsymbol{O}: \boldsymbol{O O}^{T}=\boldsymbol{I}$, where the $T$ stands for transpose and $\boldsymbol{I}$ is the identity matrix.

From Eq. (3), it is clear how the term providing the acceleration of a generic dwarf can be evaluated, $\ddot{\boldsymbol{x}}-\boldsymbol{A}$. The acceleration of a dwarf galaxy in $S_{0}$ is due to three contributions: the gradient in the external potential $\nabla \Phi_{\text {ext }}$, the gradient in the MW potential $\nabla \Phi_{\mathrm{MW}}$, and the gradient in the M 31 potential, $\nabla \Phi_{\mathrm{M} 31}$, i.e.,

$\ddot{x}=-\nabla_{x}\left(\Phi_{\text {ext }}+\Phi_{\mathrm{MW}}+\Phi_{\mathrm{M} 31}\right)$.

After applying a Taylor expansion to $\Phi_{\text {ext }}$ to second order around the comoving barycenter, and applying the usual definition of the tidal tensor given in Eq. (1), we can write

$$
\begin{array}{r}
\Phi_{\mathrm{ext}}(\boldsymbol{x}) \simeq \Phi_{\mathrm{ext}}(\boldsymbol{R})+\left\langle\nabla_{\boldsymbol{R}} \Phi_{\mathrm{ext}}(\boldsymbol{R}), \boldsymbol{x}-\boldsymbol{R}\right\rangle \\
-\frac{1}{2}\left\langle\boldsymbol{T}_{\mathrm{ext}}(\boldsymbol{R})(\boldsymbol{x}-\boldsymbol{R}), \boldsymbol{x}-\boldsymbol{R}\right\rangle
\end{array}
$$

together with two other similar relations for the potentials of MW and M31 that are not given here for the sake of brevity, with $\langle.,$.$\rangle expressing the standard inner product. Inserting these$ three relations in Eq. (4), after some simplifications we obtain the acceleration $\ddot{\boldsymbol{x}}-\boldsymbol{A}$ in linear approximation:

$\ddot{\boldsymbol{x}}-\boldsymbol{A} \simeq\left\langle\boldsymbol{T}_{\mathrm{ext}}(\boldsymbol{R})+\boldsymbol{T}_{\mathrm{MW}}(\boldsymbol{R})+\boldsymbol{T}_{\mathrm{M} 31}(\boldsymbol{R}), \boldsymbol{x}-\boldsymbol{R}\right\rangle$.

By introducing the TTT as defined in the preceding section with

$\boldsymbol{T}(\boldsymbol{R})=\boldsymbol{T}_{\mathrm{ext}}(\boldsymbol{R})+\boldsymbol{T}_{\mathrm{MW}}(\boldsymbol{R})+\boldsymbol{T}_{\mathrm{M} 31}(\boldsymbol{R})$

and using $\boldsymbol{x}-\boldsymbol{R}=\boldsymbol{O} \boldsymbol{\xi}$, we can simplify Eq. (3), which becomes

$\ddot{\boldsymbol{\xi}}=\boldsymbol{O}^{T} \boldsymbol{T} \boldsymbol{O} \boldsymbol{\xi}-2 \boldsymbol{\Omega} \times \dot{\boldsymbol{\xi}}-\dot{\boldsymbol{\Omega}} \times \boldsymbol{\xi}-\boldsymbol{\Omega} \times(\boldsymbol{\Omega} \times \boldsymbol{\xi})$,

where we have dropped the explicit dependence on the position of the tidal tensor $\boldsymbol{T}$. This tensor is always evaluated at the barycenter $\boldsymbol{T}=\boldsymbol{T}(\boldsymbol{R})$ if not specified otherwise.

\subsection{Energy states of equilibrium}

Our aim now is to understand whether the equation of motion in Eq. (6) can lead to stable equilibrium configurations. There are several techniques for attacking this problem based on the integration of the force or the impulse over a time interval (see e.g., Binney \& Tremaine (1987, Chap. 7), the elegant Lagrangian treatment of Gnedin et al. (1999), or the sophisticated analysis in the action space of Weinberg (1994a,b).

To proceed further, we identify for the energy equilibrium configurations and the stability of a system governed by the equations of motion Eq. (6). Here, we take advantage of being able to follow the evolution of the angular velocity of the noninertial reference frame by looking at the motion on the sky of the eigenvector of the tidal tensor as already achieved in previous sections for the quadrupole (see Fig. 2).

It can be demonstrated that the Lagrangian leading to Eq. (6), up to a total derivative, can be written as

$L=\frac{m}{2}\|\dot{\xi}\|^{2}+m\langle\dot{\boldsymbol{\xi}}, \boldsymbol{\Omega} \times \boldsymbol{\xi}\rangle+\frac{m}{2}\|\boldsymbol{\Omega} \times \boldsymbol{\xi}\|^{2}-m\langle\boldsymbol{A}, \boldsymbol{O} \boldsymbol{\xi}\rangle-W$

(see for instance Landau \& Lifshitz 1969). Therefore, taking the linear momentum $\boldsymbol{p}=\frac{\partial L}{\partial \dot{\xi}}=m \dot{\boldsymbol{\xi}}+m(\boldsymbol{\Omega} \times \boldsymbol{\xi})$, we can evaluate the energy $E=\langle\boldsymbol{p}, \dot{\boldsymbol{\xi}}\rangle-L$ to be

$E=\frac{m}{2}\|\dot{\xi}\|^{2}-\frac{m}{2}\|\boldsymbol{\Omega} \times \boldsymbol{\xi}\|^{2}+m\langle\boldsymbol{A}, \boldsymbol{O} \boldsymbol{\xi}\rangle+W$.

Taking the derivative with respect to the positions, we obtain

$\frac{\partial E}{\partial \boldsymbol{\xi}}=m \boldsymbol{\Omega} \times(\boldsymbol{\Omega} \times \boldsymbol{\xi})-m \boldsymbol{O}^{T} \boldsymbol{T} \boldsymbol{\xi}$.

The equilibrium energy is then given by the solution of the equation

$\mathbf{\Omega} \times(\mathbf{\Omega} \times \xi)-\boldsymbol{O}^{T} \boldsymbol{T O} \boldsymbol{\xi}=0$.

In the reference frame $S_{1}$, by definition the external tidal tensor is always diagonal, i.e., $\boldsymbol{O}^{T} \boldsymbol{T}_{\text {ext }}(\boldsymbol{R}) \boldsymbol{O}$ is greatly simplified but, in contrast, the first term of Eq. (8) has a complicated structure and the same occurs for the other two terms defining $\boldsymbol{T}$. This way of proceeding does not allow significant simplifications but a clearer insight can be gained by moving to a new reference frame, $S_{2}$, with the same origin as the previous reference system $S_{1}$, in which the matrices representing the tidal tensors $\boldsymbol{T}_{\mathrm{M} 31}$ and $\boldsymbol{T}_{\mathrm{MW}}$ are both in diagonal form, but where $\boldsymbol{T}_{\mathrm{ext}}$ is not. From the analysis of the orbit evolution expected in a statistical interpretation of the Minimum Action, PC07 showed that MW and M 31 are roughly coplanar to the external force field, at least during the past 9 Gyr. Therefore, in our simple model we can assume that the angular velocity vector $\boldsymbol{\Omega}_{\xi_{3}}$ with which we describe the rotation of the system of reference $S_{1}$, which we recall here has its third axis $\xi_{3}$ that is parallel to the normal $\hat{\boldsymbol{n}}(\pi)$, has to be parallel to the angular-velocity vector in this new system of reference $\left(O, \hat{\boldsymbol{e}}_{\eta_{1}}, \hat{\boldsymbol{e}}_{\eta_{2}}, \hat{\boldsymbol{e}}_{\eta_{3}}\right)$, namely $\boldsymbol{\Omega}_{\eta_{3}}\left(S_{2}\right)$, that we assume rotating around its third axis $\eta_{3}$, i.e., $\boldsymbol{\Omega}_{\eta_{3}}\left(S_{2}\right) \| \boldsymbol{\Omega}_{\xi_{3}}\left(S_{1}\right)$ over the past 9 Gyr. This means that we do not allow the orbital plane of M 31 and MW to tilt with respect to the plane $\tau_{\lambda_{3}} \cong \pi$ orthogonal to the eigenvector corresponding to the most negative eigenvalue $\lambda_{3}(t)$ in the course of time evolution. Even though this assumption is fulfilled automatically in the analysis below, before looking at the evolution of the MW and M 31 in the sky centered on the LG barycenter, we have verified that the angular distance between the position vectors of MW or M31 and 
$\hat{\boldsymbol{n}}\left(\tau_{\lambda_{3}}\right)$ is $90^{\circ} \pm 3^{\circ}$, which indirectly shows that the propagation of numerical errors in the calculation of the temporal evolution of the eigenvectors is small.

On the basis of these considerations, we can suppose that $\exists \boldsymbol{N}=\boldsymbol{N}(t): \boldsymbol{\xi}=\boldsymbol{N} \boldsymbol{\eta}$, i.e., there exists a linear operator represented by a rotational matrix $N \in S O(3)$ such that the generic position vector of a dwarf galaxy in $S_{1}, \xi$, can be written as a function of the generic position vector in $S_{2}, \eta$, and the tidal tensor of the external potential can be written as:

$$
\begin{aligned}
\boldsymbol{O}^{T} \boldsymbol{T}_{\mathrm{ext}} \boldsymbol{O N} \boldsymbol{\eta} & =\left(\begin{array}{ccc}
\lambda_{1} & 0 & 0 \\
0 & \lambda_{2} & 0 \\
0 & 0 & \lambda_{3}
\end{array}\right)\left(\begin{array}{ccc}
N_{11} & N_{12} & 0 \\
N_{21} & N_{22} & 0 \\
0 & 0 & 1
\end{array}\right)\left(\begin{array}{l}
\eta_{1} \\
\eta_{2} \\
\eta_{3}
\end{array}\right) \\
& =\left(\begin{array}{c}
N_{11} \eta_{1} \lambda_{1}+N_{12} \eta_{2} \lambda_{1} \\
N_{21} \eta_{1} \lambda_{2}+N_{22} \eta_{2} \lambda_{2} \\
\eta_{3} \lambda_{3}
\end{array}\right)
\end{aligned}
$$

where we have adopted a rotation matrix spinning about $\Omega_{\eta_{3}}$. As we can see, no explicit dependence on the rotational coefficients of the matrix $\boldsymbol{O}$ is necessary in the reference system $S_{2}$ that we have chosen. However, the reader should keep in mind that $\boldsymbol{O}(t) \neq \boldsymbol{N}(t) \forall t$ and only in this new reference system $S_{2}$ have we been able to simplify the matrix as above. The general form of the rotational matrix could be casted as a combination of trigonometric functions, but this would be superfluous here. The only important thing to note is that even if the assumption of collinearity between the axis of rotation of $S_{1}$ and $S_{2}$ has been justified, nothing can be said about the moduli of their angular velocities. We cannot assume that the rotation of the pair MW and M31 or, equivalently, the reference frame tightened to this rotation spins with the same rotational velocity of the reference frame attached to the external potential! This would lead to incorrect or paradoxical results that need to be avoided. In other words, we cannot assume that $\left\|\boldsymbol{\Omega}_{\eta_{3}}\left(S_{2}\right)\right\|=\left\|\boldsymbol{\Omega}_{\xi_{3}}\left(S_{1}\right)\right\|$, which is wrong, but simply adopt the condition on the directions

$$
\frac{\boldsymbol{\Omega}_{\eta_{3}}\left(S_{2}\right)}{\left\|\boldsymbol{\Omega}_{\eta_{3}}\left(S_{2}\right)\right\|}=\frac{\boldsymbol{\Omega}_{\xi_{3}}\left(S_{1}\right)}{\left\|\boldsymbol{\Omega}_{\xi_{3}}\left(S_{1}\right)\right\|} \text {. }
$$

For example, we can explicitly write the matrix $N$ as

$\left(\begin{array}{ccc}N_{11} & N_{12} & 0 \\ N_{21} & N_{22} & 0 \\ 0 & 0 & 1\end{array}\right)=\left(\begin{array}{ccc}\cos \gamma & -\sin \gamma & 0 \\ \sin \gamma & \cos \gamma & 0 \\ 0 & 0 & 1\end{array}\right)$

where $\gamma=\gamma(t)$ is the angle between the two systems. It will change as a function of time due to the time dependence of the angular velocity of the two frames

$\gamma=\gamma\left(\Omega_{\xi_{3}}^{S_{1}}(t), \Omega_{\eta_{3}}^{S_{2}}(t)\right)$.

This also means that the angular velocity is not related to the angular momentum in a straightforward way. It will indeed be the result of the combined action of the centrifugal force due to the rotation of the frame tightened to the external force field, i.e., $\boldsymbol{T}_{\text {ext }}$, and the centrifugal force due to the rotation of the frame tightened to the motion of M 31 and MW via $\boldsymbol{T}_{\mathrm{MW}}$ and $\boldsymbol{T}_{\mathrm{M} 31}$. We have a double centrifugal effect: one caused by the external field and the other by the MW and M 31 that acts with different characteristic angular velocity (typically time-dependent angular velocities). The intensity of the force due to the centrifugal component can be written as

$$
\begin{aligned}
\| \boldsymbol{N} \Omega & \left(S_{1}\right)\left\|^{2}\right\| \boldsymbol{N} \boldsymbol{\eta}\|+\| \boldsymbol{\Omega}\left(S_{2}\right)\left\|^{2}\right\| \boldsymbol{\eta} \| \\
& =\left\|\boldsymbol{\Omega}\left(S_{1}\right)\right\|^{2}\|\boldsymbol{\eta}\|+\left\|\boldsymbol{\Omega}\left(S_{2}\right)\right\|^{2}\|\boldsymbol{\eta}\| \\
& =\left(\left\|\boldsymbol{\Omega}\left(S_{1}\right)\right\|^{2}+\left\|\boldsymbol{\Omega}\left(S_{1}\right)\right\|^{2}\right)\|\boldsymbol{\eta}\|,
\end{aligned}
$$

where $\|\boldsymbol{\eta}\|$ is the distance of the dwarf galaxy from the moving barycenter of $S_{2}$. In this frame of reference $S_{2}$, we also find that $\boldsymbol{O}^{T} \boldsymbol{T}_{\mathrm{MW}} \boldsymbol{O N} \boldsymbol{\eta}$ and $\boldsymbol{O}^{T} \boldsymbol{T}_{\mathrm{M} 31} \boldsymbol{O} \boldsymbol{N} \boldsymbol{\eta}$ are diagonal. Therefore, we immediately have (see e.g., Misner et al. 1973, Chap. 1)

$$
\boldsymbol{O}^{T} \boldsymbol{T}_{\mathrm{MW}} \boldsymbol{O} \boldsymbol{N} \boldsymbol{\eta}=\frac{G M_{\mathrm{MW}}}{\left\|\boldsymbol{\eta}_{\mathrm{MW}}\right\|^{3}}\left(\begin{array}{ccc}
-1 & 0 & 0 \\
0 & 2 & 0 \\
0 & 0 & -1
\end{array}\right)\left(\begin{array}{l}
\eta_{1} \\
\eta_{2} \\
\eta_{3}
\end{array}\right)
$$

where for simplicity we have assumed that $\hat{\boldsymbol{e}}_{\eta_{2}}$ points from the barycenter of the system toward MW, $\hat{\boldsymbol{e}}_{\eta_{3}}$ is parallel to $\Omega_{\xi_{2}}\left(S_{1}\right)$, and the third axis is oriented in such a way to form a left-handed reference frame. In the same way, we may write the analogous expression for $\boldsymbol{O}^{T} \boldsymbol{T}_{\mathrm{M} 31} \boldsymbol{O N} \boldsymbol{\eta}$. Finally, the tidal term in the condition for the energy equilibrium, Eq. (8), is given by

$\boldsymbol{O}^{T} \boldsymbol{T} \boldsymbol{O}=\left(\begin{array}{ccc}\alpha_{11} & N_{12} \lambda_{1} & 0 \\ N_{21} \lambda_{2} & \alpha_{22} & 0 \\ 0 & 0 & \alpha_{33}\end{array}\right)$

with

$$
\begin{aligned}
& \alpha_{11}=N_{11} \lambda_{1}-\frac{G M_{\mathrm{MW}}}{\left\|\eta_{\mathrm{MW}}\right\|^{3}}-\frac{G M_{\mathrm{M} 31}}{\left\|\eta_{\mathrm{M} 31}\right\|^{3}} \\
& \alpha_{22}=N_{22} \lambda_{2}+\frac{2 G M_{\mathrm{MW}}}{\left\|\eta_{\mathrm{MW}}\right\|^{3}}+\frac{2 G M_{\mathrm{M} 31}}{\left\|\eta_{\mathrm{M} 31}\right\|^{3}} \\
& \alpha_{33}=\lambda_{3}-\frac{G M_{\mathrm{MW}}}{\left\|\eta_{\mathrm{MW}}\right\|^{3}}-\frac{G M_{\mathrm{M} 31}}{\left\|\eta_{\mathrm{M} 31}\right\|^{3}},
\end{aligned}
$$

which provides a simple description of the tidal effects. The above relation can be simplified further by recalling that, from the definition of barycenter in the reference frame in use, we can write

$\eta_{2}^{\mathrm{MW}}=-\frac{\eta_{2}^{\mathrm{M} 31} M_{\mathrm{M} 31}}{M_{\mathrm{MW}}}$.

Inserting this expression into Eq. (8), written in the $S_{2}$ frame, and using the relation $\left\|\boldsymbol{\eta}_{\mathrm{MW}}\right\|=\sqrt{0+\left(\eta_{2}^{\mathrm{MW}}\right)^{2}+0}=\left|\eta_{2}^{\mathrm{MW}}\right|$ i.e. $\left\|\boldsymbol{\eta}_{\mathrm{MW}}\right\|^{3}=\left|\eta_{2}^{\mathrm{MW}}\right|^{3}$, after tedious algebraic simplifications, we obtain for the three components of the tidal energy

\section{$\boldsymbol{O}^{T} \mathbf{T O} \eta=$}

$$
=\left(\begin{array}{c}
N_{12} \lambda_{1} \eta_{2}+\eta_{1}\left(N_{11} \lambda_{1}-\frac{G\left(M_{\mathrm{MW}}^{4}+M_{\mathrm{M} 31}^{4}\right)}{M_{\mathrm{M} 31}^{3}\left|\eta_{2}^{\mathrm{M} 31}\right|^{3}}\right) \\
N_{21} \lambda_{2} \eta_{1}+\eta_{2}\left(N_{22} \lambda_{2}+\frac{2 G\left(M_{\mathrm{MW}}^{4}+M_{\mathrm{M} 31}^{4}\right)}{M_{\mathrm{M} 31}^{3}\left|\eta_{2}^{\mathrm{M} 31}\right|^{3}}\right) \\
\eta_{3}\left(\lambda_{3}-\frac{G\left(M_{\mathrm{MW}}^{4}+M_{\mathrm{M} 31}^{4}\right)}{M_{\mathrm{M} 31}^{3}\left|\eta_{2}^{\mathrm{M} 31}\right|^{3}}\right)
\end{array}\right) .
$$

In the same way, we can derive the term due to the apparent force. Switching to the revolving system $S_{2}$, we apply another rotation expressed by $\boldsymbol{N} \boldsymbol{\Omega} \times(\boldsymbol{N} \boldsymbol{\Omega} \times \boldsymbol{N} \boldsymbol{\eta})=\boldsymbol{N}(\boldsymbol{\Omega} \times(\boldsymbol{\Omega} \times \boldsymbol{\eta}))$, where $\boldsymbol{\Omega}=\boldsymbol{\Omega}\left(\boldsymbol{\Omega}\left(S_{1}\right), \boldsymbol{\Omega}\left(S_{2}\right)\right)$ is the angular velocity of the system $S_{2}$ as seen from $S_{0}$. The resulting centrifugal term is

$$
\mathbf{\Omega} \times(\boldsymbol{\Omega} \times \boldsymbol{\eta})=\left(\begin{array}{c}
-\left(N_{11} \eta_{1}+N_{12} \eta_{2}\right) \Omega_{3}^{2} \\
-\left(N_{21} \eta_{1}+N_{22} \eta_{2}\right) \Omega_{3}^{2} \\
0
\end{array}\right)
$$

The associated equilibrium energy state is given by the solution of the systems

$$
\left\{\begin{array}{l}
-N_{12} \eta_{2}\left(\lambda_{1}+\Omega_{3}^{2}\right)+\eta_{1}\left(\gamma-N_{11} \lambda_{1}-N_{11} \Omega_{3}^{2}\right)=0 \\
-2 \gamma \eta_{2}-\left(N_{21} \eta_{1}+N_{22} \eta_{2}\right)\left(\lambda_{2}+\Omega_{3}^{2}\right)=0 \\
\left(\gamma-\lambda_{3}\right) \eta_{3}=0
\end{array}\right.
$$


where

$\gamma \equiv \frac{G\left(M_{\mathrm{MW}}^{4}+M_{\mathrm{M} 31}^{4}\right)}{M_{\mathrm{M} 31}^{3}\left|\eta_{2}^{\mathrm{M} 31}\right|^{3}}>0 \quad \forall t$.

The system of equations given in Eq. (10) is the result we are looking for. It is evident from these equations that the plane $\eta_{3}=0$ is the equilibrium plane for the dynamical evolution of the gravitational system.

The above result is fully adequate for our purposes because the total potential is separated into radial and vertical components. This is a standard consequence of the linear approximation obtained by truncating the Taylor expansion of the potential at the second order. Equivalently, one could use a generating function satisfying the Stakel theorem for separability in a Hamilton-Jacobi equation for the above system (e.g. Boccaletti \& Pucacco 1998).

As far as the temporal evolution is concerned $\left.\left.(t \in] t_{\text {inf }}, t_{0}\right]\right)$, we have $\eta_{3}(t)=0$ which, translated into our spatial resolution, simply means a physical spatial resolution $\left|\eta_{3}\right|<100 \mathrm{kpc}$. Furthermore, one can never have $\left(\gamma-\lambda_{3}\right)=0$ because this would imply that $\exists t: \lambda_{3}(t)=\gamma(t)$, whereas according to the definition in Eq. (11), we have $\gamma(t)>0 \forall t$, and finally $\lambda_{3}<0 \forall t_{\mathrm{lb}}<$ 9 Gyr as shown by Fig. 2. These last two conditions are clearly inconsistent leading to a contradiction that concludes our proof as required.

Therefore, the major conclusion of this demonstration is that the only possible solution is the following one: the statistical minimization of PCO7 is compatible with a planar distribution of the dwarfs and it is not a mere coincidence. This result completes the missing interpretation of the result already included in PC07.

\subsection{Stability of the equilibrium configuration}

Finally, we examine the stability of the equilibrium plane that we have found by solving Eq. (10). We take the energy of Eq. (7) written for the system $S_{2}$

$\frac{\partial E}{\partial \boldsymbol{\eta}}=m \boldsymbol{N}[\boldsymbol{\Omega} \times(\boldsymbol{\Omega} \times \boldsymbol{\eta})]-m \boldsymbol{O}^{T} \boldsymbol{T} \mathbf{O N} \boldsymbol{\eta}$

and calculate the derivative

$\frac{\partial^{2} E}{\partial \boldsymbol{\eta} \partial \boldsymbol{\eta}}=m \frac{\partial}{\partial \eta_{g}}\left\{N_{i j} \varepsilon_{\mathrm{jk} \mathrm{l}} \Omega_{\mathrm{k}} \varepsilon_{\mathrm{lmn}} \Omega_{\mathrm{m}} \eta_{\mathrm{n}}-[\boldsymbol{\Gamma} \boldsymbol{N}]_{i j} \eta_{j}\right\}$,

where $\varepsilon_{\mathrm{ijk}}$ is the Levi-Civita symbol, summation over the repeated index is assumed and we define $[\boldsymbol{\Gamma} \boldsymbol{N}]_{i j} \equiv\left[\boldsymbol{O}^{T} \boldsymbol{T} \boldsymbol{O N}\right]_{i j}$. Indicating with $\boldsymbol{S}=\boldsymbol{O N}$ the composition of the two rotation matrices $\boldsymbol{O}$ from $S_{0}$ to $S_{1}$ and $\boldsymbol{N}$ from $S_{1}$ to $S_{2}$, after some algebraic simplifications we obtain a more compact form

$\frac{\partial^{2} E}{\partial \boldsymbol{\eta} \partial \boldsymbol{\eta}}=-m \boldsymbol{N}\left\{\boldsymbol{\Theta}+\boldsymbol{S}^{T} \mathbf{T} \boldsymbol{S}\right\}$

where we have defined another matrix

$$
\begin{aligned}
\Theta_{i j} & \equiv\|\boldsymbol{\Omega}\|^{2} \delta_{i j}-\Omega_{i} \Omega_{j} \\
& =\left(\begin{array}{ccc}
\Omega_{2}^{2}+\Omega_{3}^{2} & -\Omega_{1} \Omega_{2} & -\Omega_{1} \Omega_{3} \\
-\Omega_{2} \Omega_{1} & \Omega_{1}^{2}+\Omega_{3}^{2} & -\Omega_{2} \Omega_{3} \\
-\Omega_{3} \Omega_{1} & -\Omega_{3} \Omega_{2} & \Omega_{1}^{2}+\Omega_{2}^{2}
\end{array}\right) .
\end{aligned}
$$

This matrix has no inverse, determinant $|\Theta|=0$, and trace $\operatorname{Tr}(\Theta)=2\|\boldsymbol{\Omega}\|^{2}$.
At this stage, the usual procedure would be to solve for the eigensystem in Eq. (12). However, we can avoid this complication by noticing that in the linear approximation the vertical potential decouples from the radial one. In other words, by applying the full procedure we would obtain three eigenvalues $\left\{\alpha_{1}(t), \alpha_{2}(t), \gamma-\lambda_{3}(t)\right\}$, where $\alpha_{1}(t)$ and $\alpha_{2}(t)$ are two complicated functions of time, whereas the last eigenvalue has to be exactly the most negative eigenvalue of the external tidal field for which we have already calculated the time dependence as shown in Fig. 2. As long as the eigenvalue remains negative, the relation $\gamma(t)-\lambda_{3}(t)>0 \Leftrightarrow \gamma(t)>\lambda_{3}(t)$ holds because $\gamma>0$ by definition (Eq. (10)) whereas $\lambda_{3}<0$ as required in Fig. 2. Therefore, the plane $\pi$ is stable.

\subsection{Equations of motion and force balance}

Finally, we can obtain a much deeper insight into the physical reasons for the existence of the plane $\pi$ by analyzing the equation of motion in $S_{2}$. If the plane $\pi$ is a stable configuration of the spatial distribution of dwarf galaxies, it is important to isolate the force acting on it. The equations of motion in $S_{2}$ are, from e.g., Eq. (6),

$$
\begin{aligned}
\ddot{\boldsymbol{\eta}}= & \boldsymbol{N}^{T}\left(\left\langle\boldsymbol{T}_{\mathrm{ext}}(\boldsymbol{R})+\boldsymbol{T}_{\mathrm{MW}}(\boldsymbol{R})+\boldsymbol{T}_{\mathrm{M} 31}(\boldsymbol{R}), \boldsymbol{N} \boldsymbol{\eta}\right\rangle\right) \\
& -2 \boldsymbol{\Omega} \times \dot{\boldsymbol{\eta}}-\dot{\boldsymbol{\Omega}} \times \boldsymbol{\eta}-\boldsymbol{\Omega} \times(\boldsymbol{\Omega} \times \boldsymbol{\eta})
\end{aligned}
$$

where for the different elements of the tidal tensor we can now write

$$
\begin{aligned}
\boldsymbol{N}^{T} \boldsymbol{T}_{\mathrm{M} 31} \boldsymbol{N} \boldsymbol{\eta} & =\left(\begin{array}{ccc}
-\frac{G M_{\mathrm{M} 31} \eta_{1}^{\mathrm{M} 31}}{\left\|\eta_{\mathrm{M} 31}\right\|^{3}} & 0 & 0 \\
0 & \frac{2 G M_{\mathrm{M} 31} \eta_{2}^{\mathrm{M} 31}}{\left\|\eta_{\mathrm{M} 31}\right\|^{3}} & 0 \\
0 & 0 & -\frac{G M_{\mathrm{M} 31} \eta_{3}^{\mathrm{M} 31}}{\left\|\eta_{\mathrm{M} 31}\right\|^{3}}
\end{array}\right),
\end{aligned}
$$

an analogous equation for MW, and

$$
\boldsymbol{N}^{T} \boldsymbol{T}_{\mathrm{ext}} \boldsymbol{N}=\left(\begin{array}{ccc}
T_{11} & T_{12} & 0 \\
T_{12} & T_{22} & 0 \\
0 & 0 & \lambda_{3}
\end{array}\right) .
$$

The equilibrium in the $\pi$ plane is given by

$$
\left\{\begin{array}{l}
\left(T_{11}-\frac{G M_{\mathrm{M} 31}}{\left\|\eta_{\mathrm{M} 31}\right\|^{3}}-\frac{G M_{\mathrm{MW}}}{\left\|\eta_{\mathrm{MW}}\right\|^{3}}\right) \eta_{1} \\
+T_{12} \eta_{2}+\eta_{1} \Omega_{3}^{2}-2 \Omega_{3} \dot{\eta}_{2}+\dot{\Omega}_{3} \eta_{2}=0 \\
T_{12} \eta_{1}+\left(T_{22}+\frac{2 G M_{\mathrm{M} 31}}{\left\|\eta_{\mathrm{M} 31}\right\|^{3}}+\frac{2 G M_{\mathrm{MW}}}{\left\|\eta_{\mathrm{MW}}\right\|^{3}}\right) \eta_{2} \\
+\eta_{2} \Omega_{3}^{2}+2 \Omega_{3} \dot{\eta}_{1}-\Omega_{3} \eta_{1}=0 \\
\eta_{3}\left(-\frac{G M_{\mathrm{M} 31}}{\left\|\eta_{\mathrm{M} 31}\right\|^{3}}-\frac{G M_{\mathrm{MW}}}{\left\|\eta_{\mathrm{MW}}\right\|^{3}}+\lambda_{3}\right)=0,
\end{array}\right.
$$

which sheds light on what is happening in reality ${ }^{3}$

Along the direction orthogonal to the plane $\pi$, three forces are present. One is due to the MW, $-\frac{G M_{\mathrm{MW}}}{\left\|\eta_{\mathrm{MW}}\right\|^{3}}$, another due to M 31 , $-\frac{G M_{\mathrm{M} 31}}{\left\|\eta_{\mathrm{M} 31}\right\|^{3}}$, and the third one due to the external field $+\lambda_{3}<0$. They all accumulate and act by influencing the initial motion conditions of the proto-dwarf galaxies and by preventing the dwarfs to escape from the equilibrium positions within the plane. Therefore these forces are responsible for the flattening of the

\footnotetext{
3 Here we did not use the Eq. (9) previously necessary for the energy analysis, in favor of a clearer and easier physical interpretation of the terms in the equation.
} 
whole system. This tendency of flattening the distribution of dwarf galaxies, and ensuring the stability of the plane $\pi$, can be considered valid in the limit of the linear approximation, i.e., roughly for $150 \mathrm{kpc}$ above and below the plane and for a period of time of roughly $9 \mathrm{Gyr}$, thus being partially able to imprint the initial proper motions of the dwarf galaxies taken into consideration for the Local Group.

Along the directions parallel to the plane, the situation is more complicated and described by relations such as

$$
\begin{aligned}
\left(T_{11}\right. & \left.-\frac{G M_{\mathrm{M} 31}}{\left\|\eta_{\mathrm{M} 31}\right\|^{3}}-\frac{G M_{\mathrm{MW}}}{\left\|\eta_{\mathrm{MW}}\right\|^{3}}\right) \eta_{1} \\
& +T_{12} \eta_{2}+\eta_{1} \Omega_{3}^{2}-2 \Omega_{3} \dot{\eta}_{2}+\dot{\Omega}_{3} \eta_{2}=0 .
\end{aligned}
$$

The first two terms in the sum on the left hand side are due to the tidal field that has to balance the third term due to the centrifugal force, the last term is due to the Coriolis effect and the extra term is derived from a non-uniform rotation of the system $S_{2}$. The same kind of relation holds for the other coordinates.

\section{Summary, conclusions, and consideration about the limits of the approach used}

The spatial distribution of the galaxies in the LG is the footprint of its formation mechanism, the internal gravitational interactions among the galaxies, and the gravitational action of external massive galaxies or galaxy groups on the LG members.

In this paper, we have thoroughly addressed the whole subject focusing attention on the role played by the tidal force field exerted by external galaxies or galaxy groups on the dwarf galaxies of the LG, excluding those that are clearly under the dominant gravitational effects of the HGs, in shaping the large-scale distribution of the LG galaxies.

The idea stands on the well-known effect of tidal interactions, which can be expressed as a function of the gradient in the gravitational force. While the gravitational force never changes sign, its gradient can do so. Moreover, while the gravitational force field at any distance from the center of mass of a system depends only on the inner distribution of matter, the tidal force field does not; it is indeed the result of both internal and external distributions of matter. The tidal force acting on a body moving along a certain direction will pull it away from the origin of the reference frame and, at the same time, push it along directions perpendicular to the motion toward the origin of the reference frame. Therefore, a system subjected to tidal interactions can undergo not only tidal stripping but also tidal compression. In other words, the space distribution of galaxies undergoing tidal interactions tends to become flat.

The results of this study can be summarized as follows:

- The tidal forces can be the cause of the planar distribution of these dwarf galaxies. We analytically obtain the same numerical results as Pasetto \& Chiosi (2007). We prove that a planar distribution of all dwarf galaxies, excluding those tightly bounded to a $\mathrm{HG}$, is compatible with the presence of an external force field.

- The planar geometrical distribution found by Pasetto \& Chiosi (2007) was not known to relate to the most negative eigenvalue (and associated eigenvector) of the tidal tensor. In this respect, our previous work was partially incomplete. Here we have studied this issue further by following the original idea of Raychaudhury \& Lynden-Bell (1989) to check whether the planar distribution is a mere coincidence or the consequence of fundamental laws of mechanics. To address this, we first check, using different arguments, the coincidence between the direction given by the vector orthogonal to the geometrical plane and that corresponding to the eigenvector with the most negative eigenvalue. Second, we analyze the energy of the orbital motion of the LG galaxies and find that the minimum energy corresponds to a planar distribution that correspond exactly to the geometrical plane $\pi$. Therefore, the planar distribution is the consequence of the long time-scale influence of the tidal forces exerted by massive galaxies or galaxy groups external to the LG. Finally, we demonstrate that this situation has been stable over the last 9 Gyr.

- The equilibrium and stability of the plane is a consequence of the minimum in the Action and of the orbits that come from this minimum. Although Pasetto \& Chiosi (2007) have carefully investigated the nature of the Minimum Action (whether local or absolute), the uncertainty affecting the orbits derived from the Action minimization could lead to an uncertainty in the energy analysis, the stability of the plane, and the compression effectas we have demonstrated here. This problem is still unsolved and cannot be resolved at the present time because higher quality observational data would be required (e.g., proper motions, velocities, distance moduli, absolute positions, masses). Hence, the analytical approach presented here still requires further support from independent arguments. Along this line of thought, we note that the study of van der Marel \& Guhathakurta (2008) found compatibility between their results what in Pasetto \& Chiosi (2007).

The completeness of the sample of external galaxies listed in Table 1 is another factor influencing results developed here as well as in Pasetto \& Chiosi (2007). In the previous paper, special care was taken to confirm the results based on the minimization of the action with an extended catalog from Peebles et al. (2001). We can also confirm an excellent concordance between the direction of the quadrupole tensor eigenvectors in Raychaudhury \& Lynden-Bell (1989) and that derived here with the independent catalog compilation of Peebles et al. (2001). Nevertheless the continuous discoveries of new LG members suggest that the true census of the LG galaxies cannot yet be considered complete (e.g., Loeb \& Narayan 2008).

- The minimum action and the study of the equilibrium by means of the first derivative of the energy of the system is a method that can be used to constrain the energy of dwarf galaxies with unknown proper motions. The missing proper motion prevents us from being able to obtain the true energy of any given dwarf, but the minimization of the action, together with the study of the tidal tensor, enable us to evaluate the derivative of the energy in this particular situation.

- We can find another example of a flat structure in the supergalactic plane, a slab of roughly $\cong 25 \mathrm{Mpc}$ in thickness and of a diameter greater than $110 \mathrm{Mpc}$ (Lahav et al. 2000), which was already proposed by de Vaucouleurs in 1953 (de Vaucouleurs 1953). All the groups of galaxies used here (Table 1) lie within this plane. With respect to this supergalactic plane the normal of the plane $\pi$ has a direction $(S G L, S G B)=\left(95^{\circ}, 69^{\circ}\right)$. This plane was determined by assuming that the vector joining the MW and M31 explicitly belongs to $\pi$. If you attempt to identify the best fit solution of the plane, say $\tilde{\pi}$, for the overall sample of the LG galaxies, without forcing the MW and M31 to belong to 
such a plane, we have, from PC07, $(l, b)=\left(46^{\circ}, 29^{\circ}\right)$, i.e., $(S G L, S G B)=\left(93^{\circ}, 67^{\circ}\right)$, for the normal to the plane $\tilde{\pi}$. Thus, this is even closer to the direction of the vector associated with the most negative eigenvalue of the tidal tensor but slightly more distant from the direction of the supergalactic North Pole. The proximity of the direction of the normal to $\pi$ and the supergalactic North Pole cannot yet be claimed as a significant result without further investigation. However, it is also not yet possible to study this problem fully, given the incompleteness of the catalogue of nearby galaxies (see Karachentsev et al. 2004). The mass estimation together with the distances for the systems involved are still the major source of uncertainty that we considered. Finally, in none of the external groups taken into account in Table 1 can we claim the existence of a similar flat distribution of dwarf galaxies that could be a first indication of a common external effect acting on all these groups. It seems that the local dwarf galaxies are primarily influenced by the external potential of nearby groups, not by the higher and more distant mass accumulation responsible for the supergalactic plane.

- The issue of the spatial distribution of nearby satellite galaxies bound to their HG remains to be addressed. These dwarf galaxies have orbits whose typical distance lies inside the dark matter halo of the hosting galaxy. Many $N$-body simulations have shown that galaxies in close proximity to a HG are affected by strong collisional interactions. The satellite dwarf galaxies may experience kick-off and interactions, and be absorbed into the halo of the HG (MW and M 31 in our case), and may abruptly change the phase-space density distribution function. In other words, the large-scale description of the gravitational interaction adopted here and in Pasetto \& Chiosi (2007) is ineffective on the short distance scales of the HG-dwarf satellite interactions. This will be the subject of a forthcoming study, in which simulations of the interaction between a HG and its satellites will be investigated in the framework of strong collisional dynamics along the line of work already initiated by Pasetto et al. (2003) and Pasetto et al. (2009).

Acknowledgements. We thank the anonymous referee for useful comments and substantial improvement in the first release of the paper. We thank E. Grebel for stimulating discussions and a critical reading of the paper. We thank S. Jin for careful reading of this manuscript. C.C. is pleased to acknowledge the hospitality and stimulating environment provided by the Max-Planck Institut für
Astrophysik in Garching during his visits in July 2007 and February 2008. This study has been financed by the University of Padua by means of a postdoc fellowship to S.P. and by EARA funds.

\section{References}

Binney, J., \& Tremaine, S. 1987, Galactic dynamics (Princeton, NJ: Princeton University Press), 747

Boccaletti, D., \& Pucacco, G. 1998, Astrophys. Lett. Commun., 35, 461

de Vaucouleurs, G. 1953, AJ, 58, 30

Dunn, A. M., \& Laflamme, R. 1993, MNRAS, 264, 865

Fusi Pecci, F., Bellazzini, M., Cacciari, C., \& Ferraro, F. R. 1995, AJ, 110, 1664

Gnedin, O. Y., Hernquist, L., \& Ostriker, J. P. 1999, ApJ, 514, 109

Grebel, E. K., Kolatt, T., \& Brandner, W. 1999, ed. P. Whitelock, \& R. Cannon, IAU Symp., 447

Hartwick, F. D. A. 2000, AJ, 119, 2248

Holmberg, E. 1969, Arkiv Astron., 5, 305

Kahn, F. D., \& Woltjer, L. 1959, ApJ, 130, 705

Kang, X., Mao, S., Gao, L., \& Jing, Y. P. 2005, A\&A, 437, 383

Karachentsev, I. D., Karachentseva, V. E., Huchtmeier, W. K., \& Makarov, D. I. 2004, AJ, 127, 2031

Koch, A., \& Grebel, E. K. 2006, AJ, 131, 1405

Kroupa, P., Theis, C., \& Boily, C. M. 2005, A\&A, 431, 517

Kunkel, W. E. 1979, ApJ, 228, 718

Lahav, O., Santiago, B. X., Webster, A. M., et al. 2000, MNRAS, 312, 166

Landau, L. D., \& Lifshitz, E. M. 1969, Mechanics 2nd edn. (Course of Theoretical Physics, Oxford: Pergamon Press)

Loeb, A., \& Narayan, R. 2008, MNRAS, 386, 2221

Lynden-Bell, D. 1983, in Internal Kinematics and Dynamics of Galaxies, ed. E. Athanassoula, IAU Symp., 100, 89

Lynden-Bell, D., \& Lynden-Bell, R. M. 1995, MNRAS, 275, 429

Metz, M., Kroupa, P., \& Jerjen, H. 2007, MNRAS, 374, 1125

Metz, M., Kroupa, P., \& Libeskind, N. I. 2008, ApJ, 680, 287

Misner, C. W., Thorne, K. S., \& Wheeler, J. A. 1973, Gravitation (San Francisco:

W.H. Freeman and Co.)

Palma, C., Majewski, S. R., \& Johnston, K. V. 2002, ApJ, 564, 736

Pasetto, S., \& Chiosi, C. 2007, A\&A, 463, 427

Pasetto, S., Chiosi, C., \& Carraro, G. 2003, A\&A, 405, 931

Pasetto, S., Grebel, E. K., Berczik, P., \& Spurzem, R. 2009, A\&A, submitted

Peebles, P. J. E. 1990, ApJ, 362, 1

Peebles, P. J. E. 1994, ApJ, 429, 43

Peebles, P. J. E., Phelps, S. D., Shaya, E. J., \& Tully, R. B. 2001, ApJ, 554, 104

Raychaudhury, S., \& Lynden-Bell, D. 1989, MNRAS, 240, 195

Sales, L., \& Lambas, D. G. 2004, MNRAS, 348, 1236

Sawa, T., \& Fujimoto, M. 2005, PASJ, 57, 429

Szebehely, V. 1967, Theory of orbits. The restricted problem of three bodies (New York: Academic Press)

van der Marel, R. P., \& Guhathakurta, P. 2008, ApJ, 678, 187

Weinberg, M. D. 1994a, AJ, 108, 1398

Weinberg, M. D. 1994b, AJ, 108, 1403

Yang, X., van den Bosch, F. C., Mo, H. J., et al. 2006, MNRAS, 369, 1293

Zentner, A. R., Kravtsov, A. V., Gnedin, O. Y., \& Klypin, A. A. 2005, ApJ, 629, 219 